\title{
TWO SHARP INEQUALITIES FOR LEHMER MEAN, IDENTRIC MEAN AND LOGARITHMIC MEAN
}

\section{Ye-Fang Qiu, MiaO-Kun Wang, Yu-Ming Chu and Gen-Di Wang}

Abstract. For $r \in \mathbb{R}$, the Lehmer mean of two positive numbers $a$ and $b$ is defined by

$$
L_{r}(a, b)=\frac{a^{r+1}+b^{r+1}}{a^{r}+b^{r}} .
$$

In this paper, we establish two sharp inequalities as follows: $I(a, b)>L_{-\frac{1}{6}}(a, b)$ and $L(a, b)>L_{-\frac{1}{3}}(a, b)$ for all $a, b>0$ with $a \neq b$. Here $I(a, b)=\frac{1}{e}\left(\frac{b^{b}}{a^{a}}\right)^{\frac{1}{b-a}}$ and $L(a, b)=$ $\frac{b-a}{\log b-\log a}$ denote the identric mean and logarithmic mean of two positive numbers $a$ and $b$ with $a \neq b$, respectively.

Mathematics subject classification (2010): 26E60.

Keywords and phrases: Lehmer mean, identric mean, logarithmic mean.

\section{REFERENCES}

[1] K. B. Stolarsky, Hölder means, Lehmer means, and $x^{-1} \log \cosh x$, J. Math. Anal. Appl., 202, 3 (1996), 810-818.

[2] Z. LiU, Remark on inequalities between Hölder and Lehmer means, J. Math. Anal. Appl., 247, 1 (2000), 309-313.

[3] E. F. Beckenbach, A class of mean value functions, Amer. Math. Monthly, 57 (1950), 1-6.

[4] E. F. Beckenbach And R. Bellman, Inequalities, Springer-Verlag, New York, 1965.

[5] Z. PÁLES, Inequalities for sums of powers, J. Math. Anal. Appl., 131, 1 (1988), 265-270.

[6] B. C. CARLson, The logarithmic mean, Amer. Math. Monthly, 79 (1972), 615-618.

[7] H. J. SEIFFERT, Ungleichungen für einen bestimmten Mittelwert, Nieuw Arch. Wisk. (4), 13, 2 (1995), 195-198.

[8] H. J. SEIFFERT, Ungleichungen für elementare Mittelwerte, Arch. Math., 64, 2 (1995), 129-131.

[9] M. K. Vamanamurthy and M. Vuorinen, Inequalities for means, J. Math. Anal. Appl., 183, 1 (1994), 155-166.

[10] J. SÁndor, On certain inequalities for means II, J. Math. Anal. Appl., 199, 2 (1996), 629-635.

[11] J. SÁNDOR, On certain inequalities for means III, Arch. Math., 76, 1 (2001), 34-40.

[12] J. SÁNDOR AND T. TRIF, Some new inequalities for means of two arguments, Int. J. Math. Math. Sci., 25, 8 (2001), 525-532.

[13] H. AlZER, Ungleichungen für Mittelwerte, Arch. Math., 47, 5 (1986), 422-426.

[14] P. S. Bullen, D. S. Mitrinović And P. M. Vasić, Means and Their Inequalities, D. Reidel Publshing Co., Dordrecht, 1988.

[15] H. AlZER, Ungleichungen für $\left(\frac{e}{a}\right)^{a}\left(\frac{b}{e}\right)^{b}$, Elem. Math., 40 (1985), 120-123.

[16] F. BURK, Notes: The geometric, logarithmic, and arithmic mean inequality, Amer. Math. Monthly, 94, 6 (1987), 527-528.

[17] T. P. LIN, The power mean and the logarithmic mean, Amer. Math. Monthly, 81 (1974), 879-883.

[18] A. O. Pittenger, Inequalities between arithmetic and logarithmic means, Univ. Beograd. Publ. Elektrotehn. Fak. Ser. Mat. Fiz., 678-715 (1980), 15-18. 
[19] A. O. Pittenger, The symmetric, logarithmic and power means, Univ. Beograd. Publ. Elektrotehn. Fak. Ser. Mat. Fiz., 678-715 (1980), 19-23. 